\title{
1 Cognitive Resilience after Prolonged Task Performance: an ERP investigation
}

3

4

5

6 Endre Takács ${ }^{\mathrm{abc}} *$, Irén Barkaszi ${ }^{\mathrm{a}}$, Anna Altbäcker ${ }^{\mathrm{a}}$, István Czigler ${ }^{\mathrm{ab}}$, László Balázs ${ }^{\mathrm{a}}$

7

8

9

10 a Institute of Cognitive Neuroscience and Psychology, Research Centre for Natural Sciences, 11 Hungarian Academy of Sciences, Budapest, Hungary

$12{ }^{\mathrm{b}}$ Institute of Psychology, Eötvös Loránd University, Budapest, Hungary

$13{ }^{\mathrm{c}}$ Doctoral School of Psychology, Eötvös Loránd University, Budapest, Hungary

14

15

16

17 Correspondence:

18 Endre Takács

19 takacs.endre@ttk.mta.hu

$20+36-1-3826816$

24 Acknowledgements

25 We would like to thank Péter Nagy for valuable contribution to data analysis and Tamás 26 Fodor for programming the VAS-F scale. 


\section{ABSTRACT}

Deleterious consequences of cognitive fatigue might be avoided if people respond with increased effort to increased demands. In this study we hypothesized that the effects of fatigue would be more pronounced in cognitive functions reflecting compensatory effort. Given that the P3a event related potential is sensitive to the direction and amount of attention allocated to a stimulus array, we reasoned that compensatory effort would manifest in increased P3a amplitudes. Therefore, we compared P3a before (Pre-test) and after (Post-test) a 2 hour long cognitively demanding (fatigue group, $n=18$ ) or undemanding task (control group, $\mathrm{n}=18$ ). Two auditory tasks, a three-stimulus novelty oddball and a duration discrimination two-choice response task were presented to elicit P3a. In the fatigue group, we used the Multi-attribute Task Battery as a fatigue-inducing task. This task draws on a broad array of attentional functions and imposed considerable workload. The control group watched mood-neutral documentary films. The fatigue manipulation was effective as subjective fatigue increased significantly in the fatigue group compared to controls. Contrary to expectations, however, fatigue failed to affect P3a in the Post-test phase. Similar null-effects were obtained for other neurobehavioral measures (P3b and behavioral performance). Results indicate that a moderate increase in subjective fatigue does not hinder cognitive functions profoundly. The lack of objective performance loss in the present study suggests that the cognitive system can be resilient against challenges instigated by demanding task performance.

Keywords:

mental fatigue, event related potentials, attention, oddball, distraction, effort 
Acute mental fatigue seems to be an inevitable experience in modern post-industrial society, as most professions require intensive mental work, while physical demands are decreasing. Mental fatigue is predictive of workplace accidents (Tucker et al. 2003) and is often hypothesized to have a detrimental effect on students' and professionals' cognitive performance in high-stakes situations (Kanfer 2011).

Acute mental fatigue can be defined as a multicomponent phenomenon with subjective, cognitive and behavioral aspects (van der Linden 2011). Subjectively mental fatigue is mainly associated with aversive states, such as lack of energy, boredom, and strain, and it typically includes a more or less explicit desire for stopping the current activity. On the behavioral level, mental fatigue is usually described as an inability to maintain performance, and it is characterized by slower and/or less accurate cognitive activity.

While people commonly report subjective fatigue even after short periods of mental exertion, behavioral fatigue is often less detectable under laboratory settings (Ackerman and Kanfer 2009). One viable explanation is that at first, fatigue appears only on the subjective level signaling that cognitive performance could be hindered. For a limited amount of time, compensatory effort can prevent adverse behavioral effects by maintaining adequate performance (Hockey 2011). Effort thus seems to be a key component in understanding mental fatigue, therefore, in this study we aimed to investigate this construct using behavioral and electrophysiological methods.

Cognitive effort can be interpreted as the individual's voluntary activation of attention in order to overcome stressors that potentially cause performance decrements (Sarter et al. 2006). Such stressors might include heightened task difficulty, sleepiness, or mental fatigue. While effort is traditionally measured by self-reported questionnaires and indicators of autonomic arousal (Venables and Fairclough 2009), it can also be associated with markers of the central nervous system. Among these, an important marker that can be administered by EEG is the P3b event related potential (ERP) component. Although the functional significance of $\mathrm{P} 3 \mathrm{~b}$ is still a matter of debate, increasing evidence support the view of $\mathrm{P} 3 \mathrm{~b}$ as the neural substrate of perceptual-cognitive decision making (Verleger et al. 2005; Kelly and O'Connell 2013). Accordingly, several studies show P3b amplitude to be correlated with the "amount of attention". For example, P3b is almost fully diminished when the subject ignores stimuli by paying attention to another task (Squires et al. 1973).

Attentional capacity can be voluntarily expanded (Esterman et al. 2014). Given the P3b's sensitivity to the amount of attentional resources, it can be hypothesized that the more attention is devoted voluntarily to task performance, the higher the P3b amplitude will be. This notion is supported by studies of Hopstaken and colleagues. They applied monotonous and slow paced but cognitively demanding tasks and found gradual decrement of P3b amplitude, indicating the waning of attentional processes potentially attributable to boredom and low task engagement. However, they managed to re-increase P3b amplitude after applying a manipulation that enhanced task engagement (Hopstaken et al. 2015a, b).

Based on these, P3b would be a perfect candidate for monitoring voluntary attentional allocation, however, there is a factor that limits its applicability. Besides being sensitive to the amount of attention, $\mathrm{P} 3 \mathrm{~b}$ is also sensitive to the degree of response certainty. If the subject is uncertain about the correctness of his/her response, either due to decreased alertness (Kelly and O'Connell 2013), or due to low detectability of the stimulus (Squires et al. 1973), the amplitude of P3b will be diminished. Therefore P $3 \mathrm{~b}$ amplitude varies unpredictably with task difficulty, depending on the balance between increasing effort and decreasing certainty (Kok 
2001). Accordingly, P3b is less suitable for monitoring compensatory attentional effort in

100 situations where compensation is no longer sufficient and task performance suffers significant impairment. Therefore, in the present study, we decided to examine compensatory effort with another component, as well. This component is the P3a, which is also thought to reflect attentional capacity.

104 P3a reflects the bottom-up process of the involuntary capture of attention, which is triggered by highly distinctive stimuli (for reviews see, Friedman et al. 2001; Escera and Corral 2007; Schomaker and Meeter 2015). Despite the fact that it reflects a bottom-up process and can be elicited in the absence of attention (Muller-Gass et al. 2007), a number of top-down effects can modulate P3a (Sussman et al., 2003; Chong et al., 2008). Similarly to P3b, an important predictor of $\mathrm{P} 3 \mathrm{a}$ is the amount of attention available. Studies have shown that the amplitude of P3a decreases considerably if the person does not pay attention to the particular stimulation (Friedman et al. 1998). Under dual-task conditions, increased task difficulty in the primary task often results in decreased P3a in the to-be ignored or secondary task (Legrain et al. 2005; Zhang et al. 2006; SanMiguel et al. 2008). Based on all of this, P3a can also be considered a sensitive indicator of the direction and amount of attention. Furthermore, the potential advantage of $\mathrm{P} 3 \mathrm{a}$ over $\mathrm{P} 3 \mathrm{~b}$ is that it is not affected by decision uncertainty, as in most experimental situations $\mathrm{P} 3 \mathrm{a}$ is elicited by a clear, distinctive stimulus.

117 Thus, in the present experiment, we intended to monitor compensatory effort evoked by mental fatigue with the use of P3a (and to a lesser extent with P3b). We hypothesized that due to mental fatigue performance will decline, P3b will change depending on the unpredictable combination of uncertainty and effort, while P3a will increase as a pure 121 reflection of effort.

122 The experiment was built on the fatigue inducing task - testing task scheme with control and experimental groups. Testing tasks were performed before and after a 2 hour Treatment phase in which the fatigue group performed a cognitively demanding task. The Multi-attribute Task Battery (MATB; Comstock and Arnegard, 1992) was applied to induce mental fatigue in the fatigue group. This multimodal task requires vigilance, auditory attention, continuous visuomotor control, and complex processing, especially planning. MATB has been reported to effectively induce subjective fatigue (Harris et al. 1995). Scholars and most participants usually label MATB “engaging” (Wilson et al. 2007), which has the added value that MATB can evoke fatigue without a high degree of boredom. During the treatment phase, members of the control group watched emotionally neutral, non-arousing documentaries.

132 Two tasks were administered to elicit P3a, so that we can reliably demonstrate that P3a is 133 sensitive to compensatory processes and not confounded by task-specific changes. One of them was a three-stimulus novelty oddball task, in which simple, frequent sounds are interspersed with rare higher simple sounds that require behavioral responses. Additionally, complex environmental noises with no response needed were infrequently presented, which are shown to reliably elicit the P3a component (Barkaszi et al. 2013). The other employed task was an auditory duration discrimination task, the so-called Distraction task, in which the appearance of an infrequent, task irrelevant stimulus feature (higher pitch) triggers P3a (Schröger and Wolff 1998). Although of secondary importance, with this task we were also able to study how mental fatigue and compensatory effort affect distractibility. In the Distraction task, responses to deviant stimuli that carry the task-irrelevant feature are typically slower and often less accurate than those to standard stimuli (referred to as distraction effect), which can be interpreted as a behavioral sign of distraction.

145 In addition to the P3a eliciting tasks, we also used a short version of the Psychomotor 146 Vigilance Task (PVT; Dinges and Powell, 1985), so that we could exclude the possibility that 
instead of inducing mental fatigue, our experimental manipulation reduced alertness. As the literature of sleep deprivation reveals, a decline in alertness impairs almost all cognitive functions, but the most significant deteriorations are observed in simple vigilance tasks, such as the PVT (Lim and Dinges 2010).

\section{MATERIALS AND METHODS}

\section{2}

153

\section{Participants}

Thirty-six paid volunteers participated in the study, 18 in the fatigue (11 female, mean age 22.17 years, range: $20-24$ years) and 18 in the control group ( 8 female, mean age 22.53 years, range: 19-28 years). According to self-report, participants were free of neurological disorders and were not using drugs that affect the central nervous system. They had normal or corrected to normal vision and normal hearing thresholds. Participants signed an informed consent prior to the experiment, which conformed to the Declaration of Helsinki and was approved by the Joint Ethical Committee of the Hungarian Psychology Institutes.

\section{Procedure}

The experiment consisted of three main sections, Pre-test, Treatment and Post-test phase (see Online Resource 1 for depiction). In the Pre- and Post-test phases both groups performed the same set of tasks. The order of tasks was fixed, with the exception that the order of the Oddball and Distraction tasks was counterbalanced. The Pre-test and Post-test phase was approximately 45-45 minutes long. During the Treatment phase, the fatigue group performed the Multi-attribute Task Battery (MATB), while the control group watched documentary films. This section was two hours long with no breaks allowed. A 10 minutes long mandatory break was scheduled after the Pre-test phase for both groups. After the completion of the Treatment phase, the Post-test phase began immediately. All participants stayed in the EEG booth for the entire duration of the experiment, except for the mandatory break. The EEG booth was moderately lit. Participants were seated in a reclining chair 1.2 meters from the computer monitor.

Participants took part in a practice session one or two weeks before the experiment, when they were familiarized with the experimental tasks. As for the full length measurement, participants were instructed to arrive at the laboratory after a full night of sleep. Caffeine intake was not allowed during the experiment, but we did not impose strict requirements on the caffeine consumption preceding the experiment (in order to avoid caffeine withdrawal effects). All measurements started at the same time of the day, at 9 a.m.

\section{Tasks and scales}

\section{Pre- and Post-test phase}

At the beginning of the Pre- and Post-test phases, fatigue was assessed with the 18 item VASF scale (Lee et al. 1991) translated to Hungarian and implemented in a computerized version. Participants responded by moving a small vertical bar along a horizontal line between two endpoints describing opposing statements (e.g. "not at all tired" vs. "extremely tired").

Fatigue assessment was followed by resting state EEG. Resting state EEG measurements (eyes closed and eyes open states) were 90-90 seconds long; the results of these conditions will not be reported here. 
Resting EEG was either followed by an Oddball or a Distraction task, given that the order of the two tasks was counterbalanced across participants. A three-stimulus auditory novelty oddball was administered (Oddball task). Frequent standards (80\%), infrequent targets (10\%), and infrequent novel (10\%) sounds were presented in pseudo-random order (i.e. targets were always followed by at least one standard). Standards were low tones (composed of a $887 \mathrm{~Hz}$ fundamental frequency and the second and third harmonics), targets were high tones $(938 \mathrm{~Hz}$ fundamental frequency and the second and third harmonics) and novel stimuli were various environmental sounds (e.g. glass breaking, engine starting, etc.). Participants were required to press a button with their dominant hand upon hearing the target sound. The duration of tones was $110 \mathrm{~ms}$ (5 ms rise and fall times).

The Distraction task was an auditory two-choice duration discrimination task (Schröger and Wolff 1998). Participants were presented with long $(400 \mathrm{~ms})$ and short $(200 \mathrm{~ms})$ tones of equal probability and were required to press buttons according to the duration of the tone. The pitch of the tones was $440 \mathrm{~Hz}$ in the majority of cases (86\%; standard tones), and $480 \mathrm{~Hz}$ in rare cases (14\%; deviant tones). The assignment of long and short tones to responding hands was counterbalanced between participants. The tones were presented in a pseudorandom order in which deviants were always followed by at least three standards. In both the Oddball and the Distraction task, the mean stimulus onset asynchrony was $1300 \mathrm{~ms}$ (jittered randomly between 1200-1400 ms). Sounds were presented binaurally via headphones, with an intensity of $60 \mathrm{~dB}$ above hearing level, individually adjusted for each participant.

We applied a shortened, 5 minute version of the classic PVT (Psychomotor Vigilance Task; (Dinges and Powell 1985). Participants were required to press a button with their dominant hand when a number counter appeared in the center of the screen. The counter displayed the elapsed time since its onset at each screen refresh interval. In case of a valid response, the reaction time in ms was displayed on the screen as feedback. The inter-stimulus interval (ISI)

\section{Treatment phase}

215 The fatigue group completed the Multi-attribute Task Battery (MATB; Comstock and

216 Arnegard, 1992) during the Treatment phase. MATB is a multitasking platform designed to mimic the activities of aircraft pilots. Four subtasks have to be performed simultaneously. In the system monitoring task, participants detect rare off-nominal changes in static and dynamic displays. In the tracking task, participants control an erratically moving circle using a gamepad joystick. In the communications task, participants hear pre-recorded radio messages resembling standard aircraft communication messages and they are expected to tune their virtual radio to the received frequency. The resource management task requires continuous control of two tanks' fuel levels. The tanks are interconnected and receive input from each other through pumps. In case any pumps fail, participants have to find alternative routes to maintain the required fuel level. For the present experiment, we created a new schedule of task activities to impose increased workload. The tracking task was continuous during the two hours, and communication messages, system monitoring changes and pump fails were frequent. At three time points, the fatigue group also completed the NASA-TLX scale (Hart and Staveland 1988) as an assessment of subjective workload (see Online 230 Resource 1).

231 The control group watched the following documentary films in fixed order: 1) Planet Earth 232 Episode 7 Great plains (2007), 2) When we left Earth: The NASA missions: The Shuttle (2008), 3) Ocean oasis (2000). The films were chosen based on being cognitively undemanding, non-arousing and mood-neutral. All films were dubbed in Hungarian. Prior to 
watching the documentaries, participants were instructed to pay attention to the films, as they might have to answer questions about them. This aimed to minimize decrements in attention during the non-arousing documentaries. The presented questions in fact were only assessing how interesting and informative the documentaries were.

\section{EEG recording}

240 EEG was recorded with a BrainAmp amplifier (Brain Products, Gilching, Germany), DC-100 $\mathrm{Hz}$, sampling rate $1000 \mathrm{~Hz}$, with active electrodes (ActiCap) on 61 cortical sites positioned according to the extended 10-20 system. Reference electrode was placed at $\mathrm{FCz}$, ground at $\mathrm{AFz}$ channel. Electro-oculogram was recorded with electrodes attached to the outer canthi of eyes and below the right eye.

\section{Data analysis}

\section{Fatigue Scale}

247 Subjective fatigue scores of the VAS-F scale were compared in a repeated measures

248 ANOVA, using the between subject factor of Group (fatigue, control group) and the within 249 subject factor of Phase (Pre-, Post-test).

\section{Behavioral measures}

Reaction time (RT) was defined as the time between stimulus onset and button press with a minimum duration of $150 \mathrm{~ms}$ in all three tasks (Oddball, Distraction and PVT task). Median of correct responses was calculated in tasks as a RT measure. In the Oddball and Distraction task, accuracy was calculated as percent of correct responses. Standards directly following targets, novels (Oddball task) or deviants (Distraction task) were excluded from the analyses of accuracy to maintain full compatibility between the analyses of behavioral and ERP data. Participants made no incorrect responses to novel stimuli in the Oddball task during the Posttest phase, therefore we omitted this variable from the analysis. In the PVT task we only report RT, as the number of misses and lapses (RTs longer than $500 \mathrm{~ms}$ ) were negligible.

Data in all tasks were compared with repeated measures ANOVAs, with the between subject factor of Group (fatigue or control group) and the following within subject factors. RT to targets in the Oddball task was analyzed with the within-subject factor of Phase (Pre-, Posttest). Accuracy in the Oddball task was compared with the within-subject factors of Phase and Stimulus (standard, target stimuli). The analysis of RT and accuracy in the Distraction task was accomplished with the within-subject factors of Phase, Deviance (standard, deviant stimuli) and Duration (long, short stimuli). Finally, the PVT task was analyzed with the within subject factor of Phase. All statistical analysis focused on interactions that involve the Group $\times$ Phase interaction in line with the a priori hypotheses. Moreover, we checked the presence of a significant distraction-effect (i.e. slower and less accurate responses to deviants than to standards) in the Distraction task with t-tests against zero. Greenhouse-Geisser correction was applied when appropriate. We report partial eta squared $\left(\mathrm{n}_{\mathrm{p}}{ }^{2}\right)$ as measure of effect size.

\section{Event Related Potentials}

274 We analyzed event related potentials (ERPs) in the Oddball and Distraction tasks. EEG 275 analysis was performed with EEGLAB (Delorme and Makeig 2004) in MATLAB 276 (Mathworks, Natick, USA). After offline 0.5-40 Hz (highpass: Kaiser window, transition 
bandwidth: $0.5 \mathrm{~Hz}$, passband deviation: $0.001 \mathrm{~Hz}$; lowpass: Kaiser window, transition bandwidth: $10 \mathrm{~Hz}$, passband deviation: $0.001 \mathrm{~Hz}$ ) bandpass filtering, noisy channels and segments affected by non-stereotyped artifacts were removed and extended independent component analysis was carried out. Resulting independent components were automatically classified to be cortical or artifactual with the MARA plugin (Winkler et al. 2011), using a threshold that a component was classified neural if the probability of being artifactual was maximum $10 \%$.

After MARA data treatment, similar number of ICs remained in the datasets across groups before and after the Treatment phase (see Online Resource 1). After resampling to $512 \mathrm{~Hz}$, missing channels were interpolated by spherical interpolation. All electrodes were rereferenced to the average of cortical electrodes. Subsequently, epochs (100 ms before and $1000 \mathrm{~ms}$ after stimulus onset) containing correct response and voltage not exceeding $+/-70$ $\mu \mathrm{V}$ at any channel were selected for each phase and stimulus type. Only standards not directly following novels, targets and deviants were selected for further analysis. The mean voltage of the -100 to $0 \mathrm{~ms}$ interval was subtracted from epochs as baseline correction. The average number of epochs included in one ERP is presented in Online Resource 1.

As deviant-minus-standard waveforms computed from long and short stimuli are typically highly similar in the Distraction task (Schröger et al. 2000), we followed the standard approach in the field and collapsed data across the stimulus length factor. Afterwards, deviant-minus-standard difference potentials were computed.

295

296

297

Amplitude measurement windows were identified using the "collapsed localizer" approach (Luck and Gaspelin 2017). The amplitude of components was measured as the mean voltage in $100 \mathrm{~ms}$ wide time windows centered around the grand-average peak latency. P3a was measured at $\mathrm{Cz}, \mathrm{P} 3 \mathrm{~b}$ at $\mathrm{Pz}$, where components reached their respective maxima. The latency of $\mathrm{P} 3 \mathrm{~b}$ in the Oddball task was measured on individual low-pass filtered (6 Hz cutoff frequency) waveforms at $\mathrm{Pz}$ channel. Latency was defined by the most positive value between 300 and $700 \mathrm{~ms}$. The statistical analysis of mean ERP amplitudes and latencies was carried out using ANOVA with factors Phase (Pre-, Post-test) and Group (fatigue, control group).

\section{Correlations}

307 An exploratory analysis investigated the correspondence between pre-post changes in P3a 308 and P3b with pre-post changes in subjective fatigue and task performance (see Online 309 Resource 1 for details).

\section{RESULTS}

\section{Fatigue scale}

312 One control group participant's data were missing, thus we report 17 datasets in that group.

313 Subjective fatigue increased more in the fatigue (from 34.44, SE: 3.09 to 51.08 SE: 2.96) than

314 in the control group (from 31.43, SE: 3.18 to 37.97 SE: 3.05), confirmed by the significant 315 Group $\times$ Phase interaction $\left(\mathrm{F}(1,33)=7.04, \mathrm{p}=0.012, \mathrm{n}_{\mathrm{p}}{ }^{2}=0.18\right)$. Post-hoc Tukey test showed 316 that the increase in fatigue level was significant only in the fatigue group $(\mathrm{p}<0.001$, control 317 group: $\mathrm{p}=0.098$ ). These results verify that the fatigue manipulation was successful.

318 The results of the NASA-TLX workload scale are presented in Online Resource 1. 
320 Table 1 and Figure 1 summarize the results of the behavioral measures (RT and accuracy) for each Pre/Post-test tasks. Summing up shortly, we obtained no statistically significant effect involving the Group $\times$ Phase interaction, revealing that the experimental manipulation (i.e. fatigue inducement) had no effect on any behavioral measures.

As the normality assumption of the ANOVA was violated to a large extent in the case of accuracy both in the Oddball and the Distraction tasks, we ensured the validity of the above findings by conducting additional non-parametric analyses (see Online Resource 1).

The distraction-effect in the Distraction task was also unaffected by the experimental manipulation. This effect was significant in the Pre-test phase: the RT advantage of standards compared to deviants (data collapsed over the Group and Duration factor) was $8.68 \mathrm{~ms}$ $\left(\mathrm{t}(35)=3.61, \mathrm{p}<0.001, \eta_{\mathrm{p}}{ }^{2}=0.27\right)$, while the accuracy advantage was $1.75 \%(\mathrm{t}(35)=3.13$, $\left.\mathrm{p}<0.01, \mathrm{\eta}_{\mathrm{p}}{ }^{2}=0.22\right)$. As the nonsignificant Group $\times$ Phase $\times$ Deviance interactions in the ANOVAs shows, the fatigue manipulation did not evoke differential changes in these effects for the Post-test phase between the groups.

\section{Event related potentials}

\section{Oddball task}

Figure 2 shows ERP waveforms and their scalp distribution in the Oddball task. Novel stimuli elicited a very early, sharp, centrally maximal P3a, with $244 \mathrm{~ms}$ peak latency at Cz. Target stimuli evoked a parietal P3b, with 422 ms peak latency on Pz. Both the P3a and P3b peak was strongly right "skewed" (i.e. had a steep gradient from left); to prevent earlier components to be included in the measurement, the measurement window was centered on the peak latency of the $6 \mathrm{~Hz}$ lowpass filtered grand-average waveform, corresponding to a 215-315 ms and 372-472 ms measurement window, respectively. Standard stimuli elicited no discernable P3a or P3b, therefore we did not perform a formal analysis of these stimuli.

Table 1 displays the results of statistical analyses of amplitudes (P3a and P3b) and latencies (P3b). We obtained no significant Group $\times$ Phase interactions on any tests, which indicates that the mental fatigue manipulation had no effect on ERPs in the Oddball task.

\section{Distraction task}

349 In this task, we concentrated on the deviant-minus-standard difference potentials depicted on 350 Figure 2. The raw standard and deviant waveforms can be found in Online Resource 1. As 351 Figure 2e and 2f illustrate, P3a was elicited in this task over frontal and central leads with 324 352 ms peak latency on $\mathrm{Cz}$.

353 The result of the statistical analysis of the P3a amplitude is also listed in Table 1. The Group

$354 \times$ Phase interaction was nonsignificant, indicating the lack of effects on P3a amplitude in this 355 task as well.

\section{Correlations}

357 We found weak and nonsignificant correlations between changes in ERPs, subjective fatigue and task performance (see Online Resource 1 for details). 
360 The primary purpose of this experiment was to investigate whether mental fatigue induces compensatory effort, which we intended to measure with the P3a ERP component. As an experimental manipulation, the fatigue group performed a demanding cognitive task, while the control group performed a light, non-demanding task. The success of the manipulation is demonstrated by the fact that the self-rated fatigue significantly increased in the fatigue group compared to the control group. However, the experimental manipulation failed to affect task performance during the Post-test phase. Event related potentials also remained preserved, even though we anticipated that mental fatigue would result in increased P3a amplitudes reflecting compensatory effort. Similarly to behavioral performance and P3a, P3b also remained unchanged. We interpret these findings as evidence that the fatigue group was able to maintain neurobehavioral performance, despite previously having been working on a cognitively demanding task for 2 hours.

372 Our result contradicts a substantial body of findings that revealed a deterioration of cognitive performance or a change in specific ERP components using either time-on-task (Lorist et al. 2000; Boksem et al. 2005, 2006, Hopstaken et al. 2015a, b; Borragán et al. 2017) or fatigue inducing task - testing task designs (Benoit et al., 2017, Experiment2; Gergelyfi et al., 2015; Kato et al., 2009; Persson et al., 2007, 2013, van der Linden et al., 2003, 2006). However, a smaller number of studies are in line with present results (Ackerman et al., 2010; Ackerman and Kanfer, 2009; Benoit et al., 2017, Experiment1; Brewer et al., 2011), as these investigators obtained intact cognitive functioning even after long and demanding task performance.

An apparent limitation of our study is that present results cannot provide a definitive answer whether A) fatigue group participants did in fact invoke compensatory effort during Post-test phase, allowing cognitive performance to be maintained, but P3a and P3b were not sensitive to these changes or B) performance was maintained without any compensatory effort. In our view, the present study is more informative in terms of factors influencing behavioral fatigue in a fatigue inducing task - testing task design. Since our experimental design was based on a series of premises, it is possible that we failed to induce significant effects in the testing tasks as some of these premises were false. In the following, we will look at these premises in more detail.

\section{0}

\section{1. premise: The fatigue manipulation created a suboptimal state for task performance}

392 We interpret the detected changes in subjective fatigue as they represent a state in which conditions for task performance are suboptimal. This idea is rooted in the view that subjective mental fatigue, similarly to other subjective feelings, for example, emotions (Oatley et al. 1992), is a function that may provide useful signals to the organism. A common assumption regarding mental fatigue is that it is a "stop-emotion" whose function is to inform the individual about the imbalance between the cost and rewards associated with task performance (Meijman 2000; van der Linden 2011). High level of subjective fatigue represents a suboptimal state for task performance, as costs are not balanced with rewards. In addition, subjective fatigue can also add to the cognitive load of the task, as the individual must repeatedly make a decision about ignoring the signal or modifying his/her behavior. Taken together, we conclude that our first premise can be considered true.

A somewhat independent question is whether the effect of our fatigue manipulation was large enough compared to other experiments. Previous studies in which the control group watched 
documentaries (Rozand et al. 2015; Benoit et al. 2017) reported significant increases in subjective fatigue, however, as these studies have not included effect size estimates, we cannot compare the magnitude of our effect to theirs.

\section{2. premise: The suboptimal state for task performance persisted long enough}

410 Our second premise was that induced state of mental fatigue persisted at least for the duration 411 of the testing tasks (45 minutes). Unfortunately, very little is known about how the brain 412 recovers from mental fatigue and few studies are available that assessed subjective fatigue 413 throughout longer periods of time after the experimental manipulation. Massar et al. (2010) 414 report that 40 minutes after the fatigue manipulation, subjective fatigue has dropped to the baseline level. During the 40 minutes, participants either listened to an oddball sequence or drove a driving simulator while the oddball sequence was played in the background. Both tasks are considered fairly easy, making the observed reduction in fatigue reasonable. In the present experiment, we did not measure subjective fatigue during or after the Post-test phase. However, in our case, it is less likely that the fatigue group recovered from fatigue in the Post-test phase, as the Distraction task is highly demanding, and the other two tasks also require a substantial amount of focused attention.

3. premise: The applied measurements are sensitive to the induced suboptimal state

424 The difficulty of the fatigue-inducing task - testing task design is that it is not enough to choose the fatigue-inducing task appropriately, but the testing task should also be sensitive enough. A variety of theoretical considerations exists concerning the selection of proper fatigue inducing task - testing task pairs. According to the domain-general idea, the fatigue effect should appear largely independent of the type of testing task (Baumeister 2002). In contrast, the domain-specific approach suggests that the more similar cognitive functions are mobilized, the more likely the transfer of fatigue is between the two tasks (Persson et al. 2007; Anguera et al. 2012).

In the present study, we followed an intermediate approach between the domain-general and domain-specific proposals, as the fatigue inducing task was not closely matched with the testing tasks regarding their cognitive domain. However, as the MATB is a multi-domain task, there was still a considerable overlap between the cognitive functions taxed by MATB and the testing tasks. Besides multimodal stimulus presentation (visual and auditory), MATB subtasks require the activation of several cognitive functions: vigilance is involved in the system monitoring task, continuous perceptuo-motor control is essential for the tracking task, auditory verbal processing is needed in the communication task, and complex information processing is activated in the resource management task. Additionally, executive functions are required for the multitasking aspect of the MATB, and for the planning and error detection in the resource management task itself. Among our testing tasks, the Distraction and Oddball tasks demand high degree of auditory attention. In the Distraction task, the deviant stimuli are able to distract attention, and frontal lobe mediated (potentially executive) functions are assumed to be necessary to avoid the involuntary capture of attention (Andrés et al. 2006). In the Oddball and the PVT tasks, vigilance is particularly required for successful task performance.

Previous studies demonstrated performance deterioration in testing tasks with a similar degree of testing task - fatigue inducing task overlap as in our experiment. Klaassen et al. 

brain teaser tasks) to induce mental fatigue. These tasks are mainly focused on executive functions, but also require an array of other cognitive functions. The testing task was a Sternberg working memory task, which mainly tests working memory maintenance. Van der Linden et al. (2006) used a modified continuous performance task to induce mental fatigue, which, according to the authors, requires working memory and sustained attention. The testing task was a prepulse inhibition task. Prepulse inhibition is a basic and automatic function, but, to some extent, can be related to executive functions. Both Klaassen et al. and Van der Linden et al. did demonstrate performance deterioration in the testing tasks, thus we can conclude that close functional overlap is not a necessary precondition for behavioral 460 fatigue effects.

\section{Cognitive resilience}

462

463

464

465

466

467

468

469

470

471

472

473

474

475

476

477

478

479

480

481

482

483

484

485

486

487

488

489

490

491

There are two main ways of interpreting our results: we either obtained no significant changes in the testing tasks due to some methodological issues, or the lack of mental fatigue induced changes represent a real phenomenon. As discussed above, however, none of our a priori assumptions proved to be false, making methodological deficiency a less plausible explanation. Thus, present results suggest that performance loss is not an inevitable consequence of subjective mental fatigue.

This interpretation is in line with the emerging view that the human cognitive system can be resilient in many ways. Despite significant chronic hypoxia, isolation and confinement, people may have preserved cognitive functions (Barkaszi et al. 2016). Participants have shown intact executive functions even after being sleep deprived for two nights (Tucker et al. 2010). In the field of fatigue, cognitive resilience is supported by studies that point out that subjective fatigue is not a direct function of working hours. A moderate amount of overtime does not lead to fatigue if it is voluntary and/or adequately compensated with rewards (i.e. time and money) (Van Der Hulst and Geurts 2001; Beckers et al. 2008). Likewise, the seminal study of Ackerman and Kanfer (2009) has shown that the high level of cognitive performance required by the SAT college admission test can be sustained for up to 5.5 hours without performance deterioration. A particularly interesting study reported fatigue manipulations on different time scales (Blain et al. 2016). Authors demonstrated that only six hour-long fatigue inducing sessions resulted in poorer testing task performance, while onehour long sessions failed to produce such effects, which suggests that cognitive resilience might be prevalent at shorter time scales. Taken together, present results support the view that in some situations we are able to preserve an adequate level of performance despite previous mental exertion and subjective fatigue.

\section{FUNDING}

This study was funded by a Hungarian Ministry of National Development grant URK10297.

\section{CONFLICT OF INTEREST STATEMENT}

The authors declare that they have no conflict of interest.

\section{REFERENCES}

Ackerman PL, Kanfer R (2009) Test length and cognitive fatigue: an empirical examination of effects on performance and test-taker reactions. J Exp Psychol Appl 15:163-181 
Ackerman PL, Kanfer R, Shapiro SW, et al (2010) Cognitive fatigue during testing: An examination of trait, time-on-task, and strategy influences. Hum Perform 23:381-402

Andrés P, Parmentier FBR, Escera C (2006) The effect of age on involuntary capture of attention by irrelevant sounds: A test of the frontal hypothesis of aging. Neuropsychologia 44:2564-2568

Anguera JA, Bernard JA, Jaeggi SM, et al (2012) The effects of working memory resource depletion and training on sensorimotor adaptation. Behav Brain Res 228:107-115

Barkaszi I, Czigler I, Balázs L (2013) Stimulus complexity effects on the event-related potentials to task-irrelevant stimuli. Biol Psychol 94:82-9

Barkaszi I, Takács E, Czigler I, Balázs L (2016) Extreme Environment Effects on Cognitive Functions: A Longitudinal Study in High Altitude in Antarctica. Front Hum Neurosci $10: 1-12$

Baumeister RF (2002) Ego Depletion and Self-Control Failure: An Energy Model of the Self's Executive Function. Self Identity 1:129-136

Beckers DGJ, Van Der Linden D, Smulders PGW, et al (2008) Voluntary or involuntary? Control over overtime and rewards for overtime in relation to fatigue and work satisfaction. Work Stress 22:33-50

Benoit C-E, Solopchuk O, Borragan G, et al (2017) Objective but not subjective fatigue increases cognitive task avoidance. bioRxiv 208322

Blain B, Hollard G, Pessiglione M (2016) Neural mechanisms underlying the impact of daylong cognitive work on economic decisions. Proc Natl Acad Sci 113:6967-6972

Boksem MAS, Meijman TF, Lorist MM (2005) Effects of mental fatigue on attention: An ERP study. Cogn Brain Res 25:107-116

Boksem MAS, Meijman TF, Lorist MM (2006) Mental fatigue, motivation and action monitoring. Biol Psychol 72:123-132

Borragán G, Slama H, Bartolomei M, Peigneux P (2017) Cognitive fatigue: A Time-based Resource-sharing account. Cortex 89:71-84

Brewer G a, Spillers GJ, McMillan B, Unsworth N (2011) Extensive performance on the antisaccade task does not lead to negative transfer. Psychon Bull Rev 18:923-9

Chong H, Riis JL, McGinnis SM, et al (2008) To ignore or explore: top-down modulation of novelty processing. J Cogn Neurosci 20:120-34

Comstock JR, Arnegard RJ (1992) The Multi-Attribute Task Battery for Human Operator Workload and Strategic Behavior Research. NASA Tech Memo

Delorme A, Makeig S (2004) EEGLAB: An open source toolbox for analysis of single-trial EEG dynamics including independent component analysis. J Neurosci Methods 134:921

Dinges DF, Powell JW (1985) Microcomputer analyses of performance on a portable, simple visual RT task during sustained operations. Behav Res Methods, Instruments, Comput 17:652-655

Escera C, Corral MJ (2007) Role of mismatch negativity and novelty-P3 in involuntary auditory attention. J Psychophysiol 21:251-264

Esterman M, Reagan A, Liu G, et al (2014) Reward reveals dissociable aspects of sustained 
Friedman D, Cycowicz YM, Gaeta H (2001) The novelty P3: An event-related brain potential (ERP) sign of the brain's evaluation of novelty. Neurosci Biobehav Rev 25:355-373

Friedman D, Kazmerski VA, Cycowicz YM (1998) Effects of aging on the novelty P3 during attend and ignore oddball tasks. Psychophysiology 35:508-520

Gergelyfi M, Jacob B, Olivier E, Zénon A (2015) Dissociation between mental fatigue and motivational state during prolonged mental activity. Front Behav Neurosci 9:176

Harris WC, Hancock PA, Erik J, et al (1995) Performance, Workload, and Fatigue Changes Associated with Automation. Int J Aviat Psychol 5:169-185

Hart SG, Staveland LE (1988) Development of NASA-TLX (Task Load Index): Results of Empirical and Theoretical Research. Adv Psychol 52:139-183

Hockey GRJ (2011) A motivational control theory of cognitive fatigue. In: Cognitive fatigue: Multidisciplinary perspectives on current research and future applications. pp 167-187

Hopstaken JF, van der Linden D, Bakker AB, Kompier MAJ (2015a) A multifaceted investigation of the link between mental fatigue and task disengagement. Psychophysiology 52:305-315

Hopstaken JF, van der Linden D, Bakker AB, Kompier MAJ (2015b) The window of my eyes: Task disengagement and mental fatigue covary with pupil dynamics. Biol Psychol 110:100-106

Kanfer R (2011) Determinants and consequences of subjective cognitive fatigue. In: Cognitive fatigue: Multidisciplinary perspectives on current research and future applications. pp 189-207

Kato Y, Endo H, Kizuka T (2009) Mental fatigue and impaired response processes: Eventrelated brain potentials in a Go/NoGo task. Int J Psychophysiol 72:204-211

Kelly SP, O'Connell RG (2013) Internal and External Influences on the Rate of Sensory Evidence Accumulation in the Human Brain. J Neurosci 33:19434-19441

Klaassen EB, Evers EAT, de Groot RHM, et al (2014) Working memory in middle-aged males: Age-related brain activation changes and cognitive fatigue effects. Biol Psychol 96:134-143

Kok A (2001) On the utility of P300 amplitude as a measure of processing capacity. Psychophysiology 38:557-577

Lee K a, Hicks G, Nino-Murcia G (1991) Validity and reliability of a scale to assess fatigue. Psychiatry Res 36:291-8

Legrain V, Bruyer R, Guérit JM, Plaghki L (2005) Involuntary orientation of attention to unattended deviant nociceptive stimuli is modulated by concomitant visual task difficulty. Evidence from laser evoked potentials. Clin Neurophysiol 116:2165-2174

Lim J, Dinges DF (2010) A meta-analysis of the impact of short-term sleep deprivation on cognitive variables. Psychol Bull 136:375-389

Lorist MM, Klein M, Nieuwenhuis S, et al (2000) Mental fatigue and task control: planning and preparation. Psychophysiology 37:614-625

Luck SJ, Gaspelin N (2017) How to get statistically significant effects in any ERP experiment (and why you shouldn't). Psychophysiology 54:146-157 
Massar SAAS, Wester AEA, Volkerts EER, Kenemans JL (2010) Manipulation specific effects of mental fatigue: Evidence from novelty processing and simulated driving. Psychophysiology 47:1119-1126

Meijman TF (2000) The theory of the STOP-Emotion: On the functionality of fatigue. Ergon Glob Qual Saf Product 45-50

Muller-Gass A, Macdonald M, Schröger E, et al (2007) Evidence for the auditory P3a reflecting an automatic process: Elicitation during highly-focused continuous visual attention. Brain Res 1170:71-78

Oatley K, V TMS, Jenkins JM (1992) Human Emotions: Function and Dysfunction. Annu Rev Psychol 43:55-85

Persson J, Larsson A, Reuter-Lorenz P a (2013) Imaging fatigue of interference control reveals the neural basis of executive resource depletion. J Cogn Neurosci 25:338-51

Persson J, Welsh KM, Jonides J, Reuter-Lorenz PA (2007) Cognitive fatigue of executive processes: Interaction between interference resolution tasks. Neuropsychologia 45:1571-1579

Rozand V, Lebon F, Papaxanthis C, Lepers R (2015) Effect of mental fatigue on speedaccuracy trade-off. Neuroscience 297:219-230

SanMiguel I, Corral M-J, Escera C (2008) When Loading Working Memory Reduces Distraction: Behavioral and Electrophysiological Evidence from an Auditory-Visual Distraction Paradigm. J Cogn Neurosci 20:1131-1145

Sarter M, Gehring WJ, Kozak R (2006) More attention must be paid: The neurobiology of attentional effort. Brain Res Rev 51:145-160

Schomaker J, Meeter M (2015) Short- and long-lasting consequences of novelty, deviance and surprise on brain and cognition. Neurosci Biobehav Rev 55:268-279

Schröger E, Giard MH, Wolff C (2000) Auditory distraction: Event-related potential and behavioral indices. Clin Neurophysiol 111:1450-1460

Schröger E, Wolff C (1998) Behavioral and electrophysiological effects of task-irrelevant sound change: A new distraction paradigm. Cogn Brain Res 7:71-87

Squires KC, Hillyard SA, Lindsay PH (1973) Vertex potentials evoked during auditory signal detection: Relation to decision criteria. Percept Psychophys 14:265-272

Sussman ES, Winkler I, Schröger E (2003) Top-down control over involuntary attention switching in the auditory modality. Psychon Bull Rev 10:630-637

Tucker AM, Whitney P, Belenky G, et al (2010) Effects of sleep deprivation on dissociated components of executive functioning. Sleep 33:47-57

Tucker P, Folkard S, Macdonald I (2003) Rest breaks and accident risk. Lancet 361:680

Van Der Hulst M, Geurts S (2001) Associations between overtime and psychological health in high and low reward jobs. Work Stress 15:227-240

van der Linden D (2011) The urge to stop: The cognitive and biological nature of acute mental fatigue. Cogn Fatigue Multidiscip Perspect Curr Res Futur Appl 149-164

van der Linden D, Frese M, Meijman TF (2003) Mental fatigue and the control of cognitive processes: effects on perseveration and planning. Acta Psychol (Amst) 113:45-65

van der Linden D, Massar SAA, Schellekens AFA, et al (2006) Disrupted sensorimotor 
619 Venables L, Fairclough SH (2009) The influence of performance feedback on goal-setting and mental effort regulation. Motiv Emot 33:63-74

621 Verleger R, Jaśkowski P, Wascher E (2005) Evidence for an integrative role of P3b in linking reaction to perception. J Psychophysiol 19:165-181

Wilson GF, Caldwell JA, Russell CA (2007) Performance and psychophysiological measures of fatigue effects on aviation related tasks of varying difficulty. Int J Aviat Psychol $17: 219-247$

Winkler I, Haufe S, Tangermann M (2011) Automatic classification of artifactual ICAcomponents for artifact removal in EEG signals. Behav Brain Funct 7:30

628 Zhang P, Chen X, Yuan P, et al (2006) The effect of visuospatial attentional load on the processing of irrelevant acoustic distractors. Neuroimage 33:715-724

630

\section{FIGURE LEGENDS}




\section{Behavioral measures}

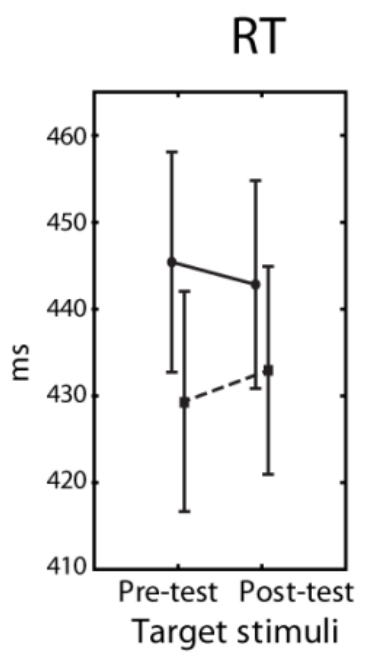

RT
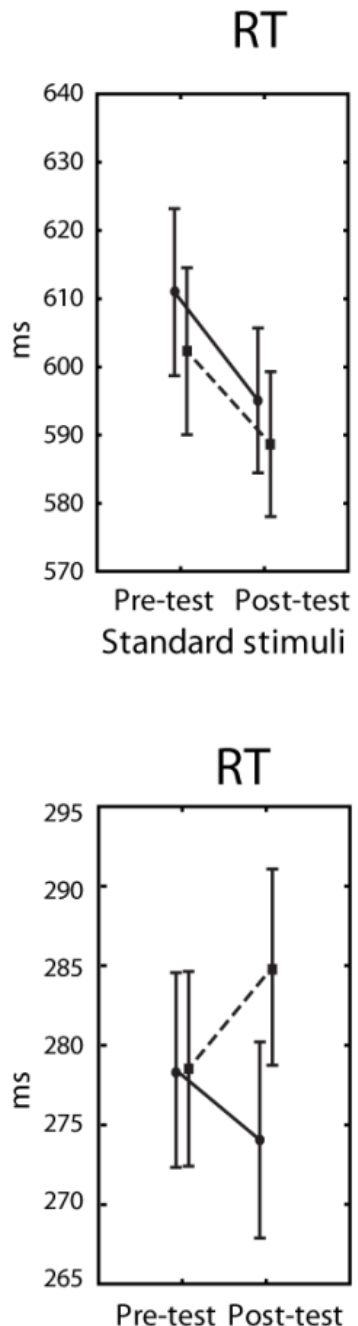

\section{Oddball task}

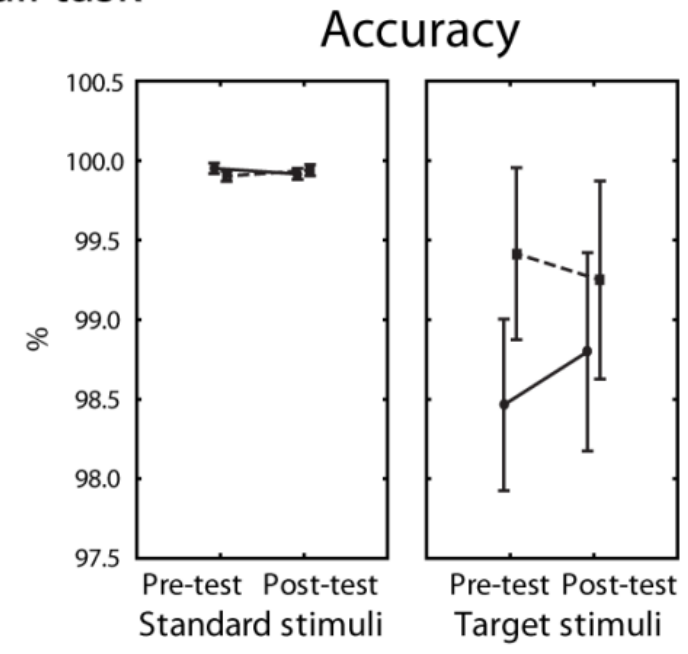

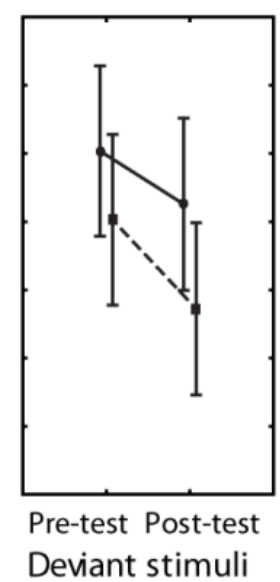
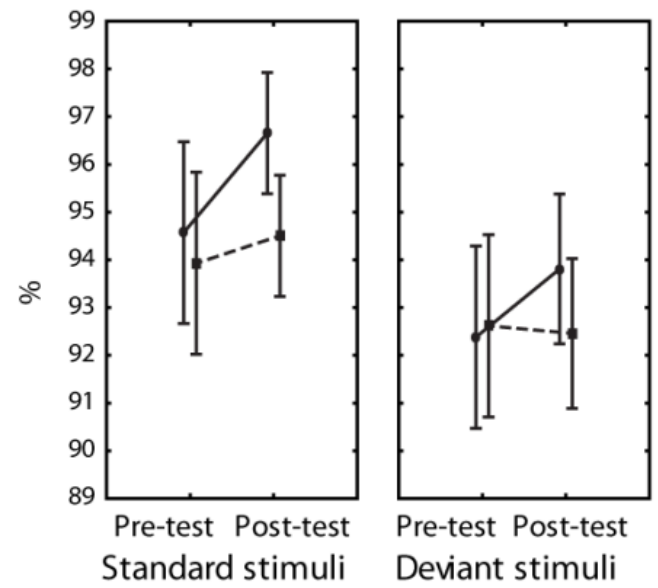

633 Fig. 1 RT and accuracy in the three Pre/Post-tasks. Vertical bars denote standard errors 
Fatigue group

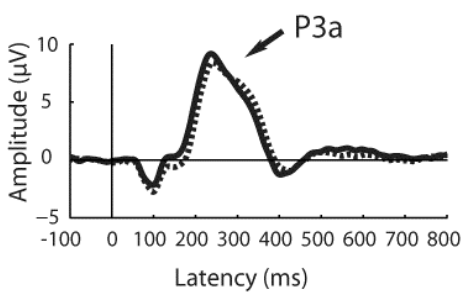

Fatigue group

b

Pre-test Post-test
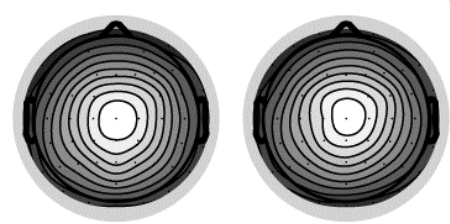

P3a 215-315 ms

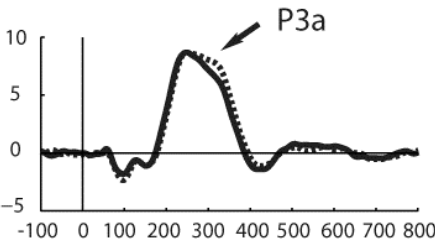

$\mathrm{Cz}$

n'?"' Pre-test - Post-test

Control group

Pre-test Post-test

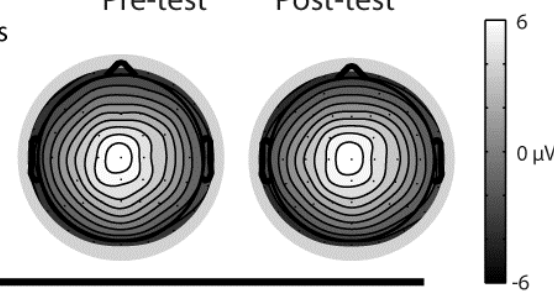

c

Oddball task, Target stimuli

Fatigue group

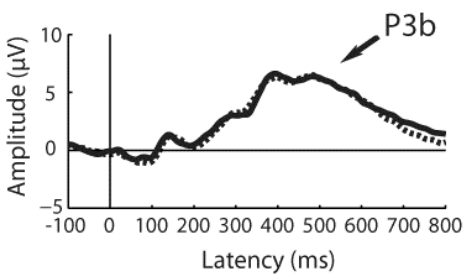

Control group

d
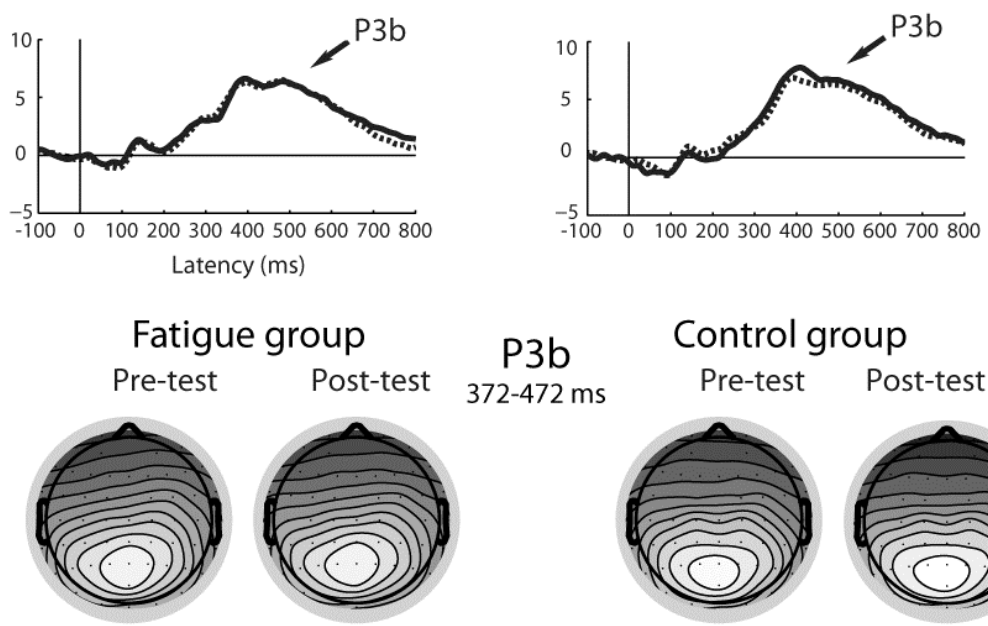

\section{Distraction task, Deviant-minus-standard \\ Fatigue group \\ Control group}

e

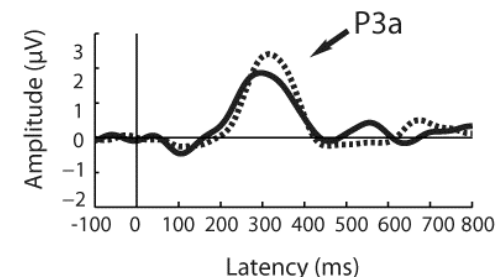

P3b Control group

372-472 ms Pre-test Post-test

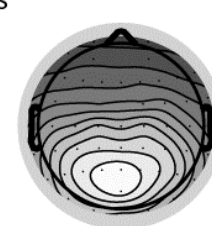

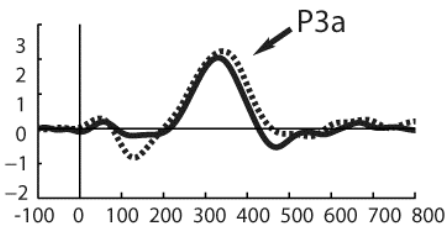

$\mathrm{Cz}$

\begin{tabular}{ll|} 
"'!"' Pre-test \\
- Post-test
\end{tabular}

f

Fatigue group
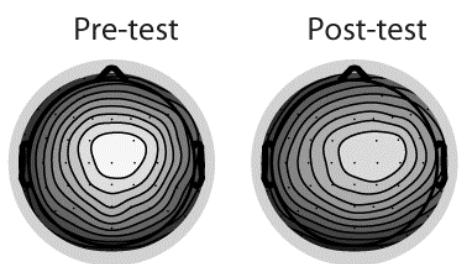

P3a

Control group

274-374 ms Pre-test
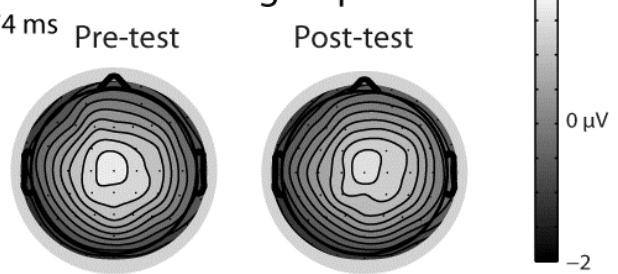
635

636

637

638

639

640

641

642

643

644

645

Fig. 2 (a) and (c) Grand-average ERPs in the Oddball task elicited by novel and target stimuli, respectively. (e) Grand-average deviant-minus-standard waveforms in the Distraction task. The waveform was low-pass filtered at $10 \mathrm{~Hz}$ for display purposes. (b) (d) and (f) Topographical distribution of ERPs

\section{TABLES}

Table 1 Statistical results for the behavioral and ERP measures in the three Pre/Post-test tasks. G: Group factor, P: Phase factor, St: Stimulus factor, D: Deviance factor, Du: Duration factor.

\begin{tabular}{|c|c|c|c|c|c|c|}
\hline Task & Measure & Effect & $\mathrm{df}$ & $\mathrm{F}$ & $\mathrm{p}$ & $\eta_{p}^{2}$ \\
\hline \multirow[t]{6}{*}{ Oddball } & RT & $\mathrm{G} \times \mathrm{P}$ & 1,34 & 0.57 & 0.46 & 0.02 \\
\hline & accuracy & $G \times P$ & 1,34 & 0.4 & 0.52 & 0.01 \\
\hline & & $\mathrm{G} \times \mathrm{P} \times \mathrm{St}$ & 1,34 & 0.7 & 0.39 & 0.02 \\
\hline & $\begin{array}{l}\text { P3a amplitude } \\
\text { (novel ERPs) }\end{array}$ & $\mathrm{G} \times \mathrm{P}$ & 1,34 & 0.69 & 0.41 & 0.02 \\
\hline & $\begin{array}{l}\text { P3b amplitude } \\
\text { (target ERPs) }\end{array}$ & $G \times P$ & 1,34 & 1.28 & 0.27 & 0.04 \\
\hline & $\begin{array}{l}\text { P3b latency } \\
\text { (target ERPs) }\end{array}$ & $\mathrm{G} \times \mathrm{P}$ & 1,34 & 0.18 & 0.67 & $<0.01$ \\
\hline \multirow[t]{9}{*}{ Distraction } & RT & $\mathrm{G} \times \mathrm{P}$ & 1,34 & 0.06 & 0.84 & $<0.01$ \\
\hline & & $G \times P \times D$ & 1,34 & 1.73 & 0.20 & 0.05 \\
\hline & & $\mathrm{G} \times \mathrm{P} \times \mathrm{Du}$ & 1,34 & 0.22 & 0.64 & 0.01 \\
\hline & & $\mathrm{G} \times \mathrm{P} \times \mathrm{D} \times \mathrm{Du}$ & 1,34 & 0.02 & 0.88 & $<0.01$ \\
\hline & accuracy & $\mathrm{G} \times \mathrm{P}$ & 1,34 & 2.47 & 0.13 & 0.07 \\
\hline & & $\mathrm{G} \times \mathrm{P} \times \mathrm{D}$ & 1,34 & 0.01 & 0.94 & $<0.01$ \\
\hline & 7 & $\mathrm{G} \times \mathrm{P} \times \mathrm{Du}$ & 1,34 & 2.68 & 0.11 & 0.07 \\
\hline & & $\mathrm{G} \times \mathrm{P} \times \mathrm{D} \times \mathrm{Du}$ & 1,34 & 2.64 & 0.11 & 0.07 \\
\hline & $\begin{array}{l}\text { P3a amplitude } \\
\text { (deviant-minus- } \\
\text { standard wave) }\end{array}$ & $\mathrm{G} \times \mathrm{P}$ & 1,34 & 0.67 & 0.42 & 0.02 \\
\hline PVT & RT & $\mathrm{G} \times \mathrm{P}$ & 1,34 & 2.87 & 0.099 & 0.08 \\
\hline
\end{tabular}

646 